\title{
Una experiencia de grupo de reflexión para el manejo de entrevistas clínicas difíciles
}

\section{A reflection group's experience in handling hard clinical encounters}

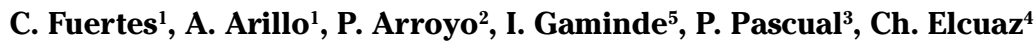

\section{RESUMEN}

Fundamento. Se presenta la experiencia de un grupo de reflexión para valorar si la metodología llevada a cabo, ayuda a los profesionales sanitarios a entender los encuentros difíciles (ED), a mejorar su satisfacción profesional y la comunicación con el paciente.

Material y método. Se siguió una metodología de reestructuración cognitiva con registro escrito para analizar, primero individualmente, los ED con los pacientes y comentarlos luego en el grupo de reflexión.

Resultados. Los ED se referían principalmente a: solicitudes vividas como inapropiadas, cuestionamiento de competencia profesional, y cambio inesperado de médico. Las emociones surgidas, antes y después de "volver a pensar", cambiaron de intensidad de forma significativa $(\mathrm{p}<0,001)$. Los pensamientos irracionales se agruparon en 9 tipos de distorsiones cognitivas, siendo las más frecuentes "razonamiento emocional" y "falacias de control". Las pautas explicativas constructivas que surgieron después de "volver a pensar", en su mayoría estaban relacionados con la necesidad de mejorar la comunicación. El grupo reconoció muchas aportaciones positivas. Ni las encuestas utilizadas para valorar el desgaste profesional (MBI), ni la encuesta diseñada para la evaluación, presentaron diferencias significativas, comparando al inicio y final del trabajo.

Conclusiones. El empleo conjunto de una metodología cognitivo-conductual en el marco de un grupo de reflexión, se complementan mutuamente, y posibilitan el trabajo en grupo sin precisar un conductor profesional. La reflexión escrita y el trabajo con los pensamientos irracionales y "volver a pensar" con la perspectiva de comentarlo en el grupo, es una experiencia aplicable en el contexto de Atención Primaria y ayuda al manejo de los encuentros difíciles.

Palabras clave. Relaciones profesional-paciente. Grupo reflexión. Atención primaria. Emociones. Encuentros difíciles.

\begin{abstract}
Background. We present the experience of a reflection group to evaluate whether the methodology carried out helps health professionals to understand difficult encounters and improve their professional satisfaction and communication with the patient.
\end{abstract}

Method. We followed a cognitive restructuring methodology with a written record in order to analyze difficult encounters with patients, first individually and then followed by comments in the reflection group.

Results. Difficult encounters mainly involved: requests felt to be inappropriate, questioning of professional competence, and unexpected change of doctor. The emotions arising, before and after "rethinking", changed in intensity in a significant way $(\mathrm{p}<0.001)$. Irrational thoughts were grouped in 9 types of cognitive distortions, with the most frequent being "emotional reasoning" and "control fallacies". The majority of constructive explanatory patterns that emerged after "rethinking" were related to the need for improving communication. The group recognized many positive contributions. Neither the surveys used to evaluate occupational burnout, nor the survey designed for the evaluation showed significant differences, comparing the beginning and end of the work.

Conclusions. The joint use of a cognitive-behavioral methodology in the framework of a reflection group is mutually complementary, and enables group work without the need for professional direction. Written reflection and work with irrational thoughts and "rethinking" with the perspective of group comment is an experience that is applicable in the context of Primary Care and helps in handling difficult encounters.

Key words. Professional-patient relationships. Focus groups. Primary health care. Emotions. Task performance and analysis.

\section{An. Sist. Sanit. Navar. 2013; 36 (3): 455-466}

1. Atención Primaria. Centro de Salud Txantrea. Pamplona.

2. Atención Primaria. Centro de Salud de Huarte.

3. Atención Primaria. Centro de Salud de Azpilagaña. Pamplona.

4. Atención Primaria. Servicio de Urgencias Extrahospitalarias. Pamplona.

5. Servicio de Investigación, Innovación y Formación Sanitaria. Departamento de Salud. Pamplona.

\section{Correspondencia: \\ Carmen Fuertes \\ Centro de Salud de Txantrea \\ $\mathrm{C} / \mathrm{M}^{\mathrm{a}}$ Auxiliadora, $\mathrm{s} / \mathrm{n}$ \\ 31015. Pamplona \\ cfuerteg@cfnavarra.es}

Recepción: 26 de julio de 2013

Aceptación provisional: 3 de septiembre de 2013

Aceptación definitiva: 4 de octubre de 2013 


\section{INTRODUCCIÓN}

La relación médico-paciente es una relación compleja, que influye en la salud de los pacientes, quienes tienen necesidades y requerimientos diferentes, como también lo son los profesionales que les atienden. El nivel de autoconocimiento del profesional sanitario repercute en el establecimiento de una relación humana de calidad ${ }^{1-3}$.

La relación médico-paciente ha sido estudiada tanto desde el punto de vista de los profesionales como de los pacientes, a través de técnicas observacionales ${ }^{4-6}$ y técnicas de entrevista ${ }^{7-9}$. Es conocido que el modo de investigación cualitativo abre un espacio al análisis en el campo de la salud que permite acercarse a las formas de comunicación y a los diferentes contextos en los que se producen las actividades de salud $^{10}$. Las investigaciones llevadas a cabo a través de grupos focales con pacientes han evidenciado la importancia de los conocimientos técnicos del profesional, así como de los elementos relacionales en información y comunicación ${ }^{11,12}$. Otros estudios han detectado que, cuando la relación profesional-paciente es conflictiva, puede comportar problemas de comunicación y sentimientos negativos ${ }^{13}$.

Hace 5 décadas se iniciaron los primeros grupos Balint y de reflexión en nuestro país. Emergió la importancia del autoconocimiento de los profesionales y de comprensión de sus propias emociones, así como de la interacción en el encuentro clínico $^{14-16}$. Los grupos de reflexión pueden servir como un medio para aumentar la satisfacción profesional y prevenir el burnout ${ }^{17-18}$. La experiencia de uno de estos grupos se presentó en un trabajo anterior $^{19}$. En dicho trabajo se constató que habían mejorado tanto la satisfacción profesional, como el desarrollo de la capacidad introspectiva de los profesionales. El grupo se planteó continuar la reflexión sobre casos, esta vez sin conductor profesional, incorporando metodología cognitivo-conductual desarrollada por diferentes autores ${ }^{20-21}$. En el presente artículo se presenta esta experiencia desarrollada durante 2 años.
Los objetivos del trabajo son:

- Investigar si la aplicación de una metodología de reestructuración cognitiva aplicada a encuentros difíciles con los pacientes y comentada en un grupo de reflexión, puede ayudar a los profesionales sanitarios a comprender mejor estas situaciones, y a mejorar consecuentemente su satisfacción profesional y sus habilidades en la comunicación con el paciente.

- Conocer y describir el tipo de emociones y pensamientos irracionales asociados que le surgen al profesional sanitario en los encuentros difíciles.

- Valorar si el hecho de analizar los casos en grupo puede significar un enriquecimiento mayor para el profesional que el análisis exclusivamente individual.

\section{MATERIAL Y MÉTODOS}

El grupo de reflexión se constituyó por 8 médicos de familia y una enfermera que habían participado los 2 años anteriores en un grupo de reflexión con conductor y continuaron otros 2 años reuniéndose mensualmente. Por turno, en cada reunión, un miembro del grupo hacía de moderadorconductor. En el grupo participaron en calidad de invitados, y durante periodos de tiempo más cortos, otros 6 médicos, sin aportar casos al grupo, pero sí participando en las discusiones.

El tipo de trabajo ha sido cuasi-experimental ("antes-después"). Se definieron como encuentros difíciles todas aquellas situaciones, que en la consulta le han producido al profesional sanitario emociones de complejo manejo.

Se utilizó un plan de análisis de emociones cognitivo-conductual, basado en la metodología de "rebatimiento" de Martin Seligman ${ }^{20}$ desarrollada en España por José Luis Bimbela y que denominó "volver a pensar" ${ }^{21}$. En el desarrollo de esta metodología, estos autores parten de la base de que la forma en que las personas se perciben y se 
sienten a sí mismas es algo que puede cambiar. Cuando cambian estas percepciones y sentimientos el efecto se extiende a todas las áreas de la vida, procurando una sensación cada vez mayor de libertad.

Con estos métodos probados de terapia conductual cognitiva, se eleva la autoestima cambiando la forma de interpretar la vida, desvelando y analizando las afirmaciones negativas que las personas hacen sobre sí mismas y creando nuevas afirmaciones, objetivas y positivas, que fomentan la autoestima en vez de socavarla.

Se trata de escuchar la "voz crítica interior" de la manera más detallada posible, para pasar posteriormente a introducir "la voz sana", a través de la capacidad de las personas de pensar en términos realistas.

Así pues el trabajo individual de cada persona participante en el grupo consistía en desarrollar la metodología siguiente, a partir de un caso vivido por ella.

Primer paso: describir el caso escuetamente y sobre todo la situación que había generado emociones en el profesional sanitario.

Segundo paso: enumerar las emociones que le habían surgido y cuantificarlas del 1 a 10 (de menor a mayor intensidad).

Tercer paso: describir los pensamientos asociados a esas emociones.

Cuarto paso: describir los nuevos pensamientos tras "pensar de nuevo", esta vez de una forma más realista y acorde a la situación.

Quinto paso: realizar una cuantificación de la variación de intensidad, o modificación de las emociones iniciales.

Toda la secuencia se anotaba en una ficha (Anexo 1) que se enviaba a la coordinadora del grupo. Si se presentaba el caso en el grupo, se anotaban posteriormente las sugerencias y aportaciones del grupo.

En cada sesión el grupo determinaba los casos que se iban a presentar, atendiendo a la urgencia que manifestaban tener los miembros del grupo interesados en presentar sus casos.
Para la evaluación se midieron los siguientes aspectos:

a. La variación en la intensidad de las emociones, así como las emociones nuevas que surgieron o desaparecieron tras la reflexión; tanto en los casos presentados en el grupo como en los que individualmente se les ha aplicado la metodología para la reconducción de emociones ${ }^{21}$. Todas las emociones se agruparon para el análisis posterior en las 5 emociones básicas que describen Ekman y coll $^{22}$ : miedo, ira, tristeza, alegría y sorpresa. Además se consideró otra emoción que algunos autores como Tracy y col $^{23}$ definen como compleja o social: "vergüenza" y que nosotros denominamos "frustración", al englobar mejor esta denominación todas las emociones agrupadas en ese epígrafe.

b. Los pensamientos irracionales que habían surgido con las emociones en cada caso. Estos se agruparon para su análisis en los 9 tipos de distorsiones cognitivas que acuñan McKay y Fanning ${ }^{24}$. También se analizaron los pensamientos surgidos tras "volver a pensar".

c. El nivel de satisfacción de los profesionales antes y después del período de trabajo del grupo, medida mediante el test $\mathrm{MBI}^{25}$ y como el MBI mide sobre todo desgaste profesional, se consideró complementarlo con una encuesta de elaboración propia (Tabla 1) sobre la satisfacción profesional ante situaciones concretas. Estas situaciones concretas se habían explicitado como relevantes y frecuentes en una experiencia anterior del grupo de reflexión ${ }^{19}$.

Se utilizaron los siguientes estadísticos: Prueba no-paramétrica test de Wilcoxon, y prueba Chi-cuadrado. El programa usado fue SPSS v20. Se valoró un nivel de significación de $\mathrm{p}<0,05$. 
Tabla 1. Resultados de la encuesta de elaboración propia para evaluar las emociones provocadas

\begin{tabular}{|c|c|c|}
\hline Situaciones y emociones provocadas & $\begin{array}{l}\text { Mediana } 2010 \\
\text { [rango] }\end{array}$ & $\begin{array}{l}\text { Mediana } 2012 \\
\text { [rango] }\end{array}$ \\
\hline 1. Ante un/a paciente dominante, tengo sensación de imposición. & $4[2]$ & $3[0]$ \\
\hline 2. Ante un/a paciente con enfermedades psicosociales, siento pereza. & $3[2]$ & $2[2]$ \\
\hline $\begin{array}{l}\text { 3. Ante un/a paciente "invasivo/a", siento incomodidad ante las preguntas } \\
\text { que me realiza. }\end{array}$ & $3[2]$ & $3[1]$ \\
\hline $\begin{array}{l}\text { 4. Ante las personas somatizadoras, siento impotencia para ayudarles a } \\
\text { comprender su malestar. }\end{array}$ & $3[2]$ & $2[2]$ \\
\hline $\begin{array}{l}\text { 5. Cuando un paciente no sigue los consejos preventivos o sobre utiliza- } \\
\text { ción de servicios, me siento utilizado/a. }\end{array}$ & $2[1]$ & $2[1]$ \\
\hline $\begin{array}{l}\text { 6. Cuando tengo una consulta con un/una paciente hiperfrecuentador/a, } \\
\text { siento rabia e impotencia. }\end{array}$ & $3[2]$ & $3[1]$ \\
\hline $\begin{array}{l}\text { 7. Ante los reproches de la familia tras algún error de diagnóstico o de } \\
\text { tratamiento, me siento culpable. }\end{array}$ & $3[3]$ & $3[3]$ \\
\hline $\begin{array}{l}\text { 8. Ante la no derivación a especialista cuando estimo posteriormente que } \\
\text { debería haberlo hecho, siento culpas y muchas dudas. }\end{array}$ & $4[2]$ & $4[3]$ \\
\hline $\begin{array}{l}\text { 9. Ante una mujer que sospecho o confirmo que ha podido ser maltratada } \\
\text { por su pareja, siento indignación y desconcierto. }\end{array}$ & $3[2]$ & $3[2]$ \\
\hline $\begin{array}{l}\text { 10. Cuando un paciente atribuye a su pareja sus problemas de salud, siento } \\
\text { rabia y dificultad para tolerar el comportamiento del paciente. }\end{array}$ & $3[2]$ & $3[2]$ \\
\hline $\begin{array}{l}\text { 11. Cuando se dan diferentes puntos de vista entre el/la paciente y sus fami- } \\
\text { liares, sobre dónde morir, siento confusión. }\end{array}$ & $3[2]$ & $3[2]$ \\
\hline $\begin{array}{l}\text { 12. Ante la petición no compartida de un/una paciente de ser derivado a } \\
\text { una especialidad, me siento poco apreciado/a profesionalmente. }\end{array}$ & $3[2]$ & $3[2]$ \\
\hline $\begin{array}{l}\text { 13. Ante un/una paciente que no se cuida suficientemente, poniendo en pe- } \\
\text { ligro su vida o la de otros (p. ej. conductor), siento malestar y confusión. }\end{array}$ & $4[2]$ & $3[3]$ \\
\hline
\end{tabular}

Nota: Se puntuaron por los 9 miembros del grupo, cada item de 1 a 5 (de nunca a siempre).

Se presenta en la tabla calculada la mediana para cada uno de los items y poder apreciar la del 2010 y 2012 sin diferencias significativas entre ambas $(\mathrm{p}>0,05)$.

\section{RESULTADOS}

Se realizaron 14 sesiones grupales a lo largo de 2 años, de 90 minutos cada una, con orientación cognitivo-conductual. En cada sesión mensual se expusieron entre 1 y 3 encuentros difíciles. Se recogieron un total de 26 casos presentados en grupo y 26 casos no presentados y trabajados a nivel individual. Al no haber encontrado diferencias en el análisis por separado de estos dos subgrupos, se presentan los resultados conjuntamente.

Las situaciones descritas reflejadas en los casos se referían a los siguientes siete aspectos: 22 de "solicitudes vividas como inapropiadas" (recetas, bajas laborales, derivaciones, informes...); 10 casos sobre "cuestionamiento de competencia profesional"; 6 de "cambio inesperado de médico"; 5 de "dilemas éticos"; 4 sobre "malas noticias"; 3 sobre "atención a familiares, amistades, colegas" y 2 de "sensación de trato incorrecto al paciente".

Todas las emociones surgidas en los encuentros, pueden verse en tabla 2. Algunas aparecían sólo al inicio del caso y otras sólo al "volver a pensar". Se valoró también la variación en la graduación de las emociones que aparecen "antes y después de volver a pensar", y en todos los casos había disminuido la intensidad de la emoción puntuada inicialmente respecto a la final. 
Tabla 2. Emociones iniciales y finales (después de "volver a pensar")

\begin{tabular}{|c|c|c|c|c|c|c|c|c|}
\hline \multicolumn{3}{|l|}{ Miedo } & \multicolumn{3}{|c|}{ Ira } & \multicolumn{3}{|c|}{ Frustración } \\
\hline & Inicial & Final & & Inicial & Final & & Inicial & Final \\
\hline ACUSADA/O & & 1 & CABREO & 1 & & ABANDONADA & & 1 \\
\hline Agredido/a & 1 & 2 & Enfado & 21 & 14 & Desprecio & 2 & 2 \\
\hline BAJA AUTOESTIMA & 4 & & Exigido & 1 & 2 & Engañado/a & 2 & 1 \\
\hline Bloqueo & 1 & 1 & NO APOYO & 1 & & Envidia & 1 & 2 \\
\hline BOCHORNO & 1 & & Fastidio & 5 & 5 & Frustración & 20 & 14 \\
\hline Celos & 1 & 3 & Ira & 3 & 2 & IMPOTENCIA & & 6 \\
\hline Confusión & 6 & 2 & Maltratado & 5 & 2 & Indiferencia & 13 & 1 \\
\hline Cuestionado/a & 1 & 3 & MENTIRA & 1 & & Insatisfacción & 1 & 2 \\
\hline Desasosiego & 2 & 1 & Molesta/o & 1 & 1 & Rechazo & 1 & 1 \\
\hline Duda & 2 & 2 & Presionado & 5 & 4 & Tonto & 1 & 1 \\
\hline Estupor & 4 & 1 & Provocado/a & 1 & 1 & TRAICIÓN & 1 & \\
\hline IMPACIENCIA & 3 & & Rabia & 14 & 6 & Arrepentimiento & 1 & 1 \\
\hline Incapacidad & 1 & 1 & Sermoneada & 1 & 1 & Culpa & 1 & 5 \\
\hline Incertidumbre & 2 & 2 & TENSION & 1 & & Vergüenza & 7 & 2 \\
\hline Incomodidad & 5 & 4 & Utilizado/a & 16 & 9 & Resignación & 3 & 2 \\
\hline Inquietud & 1 & 3 & & & & DESCONTROL & & 1 \\
\hline Inseguridad & 5 & 2 & & & & DISGUSTO & & 2 \\
\hline Intranquilidad & 4 & 1 & & & & & & \\
\hline LUCHA INTERNA & 1 & & & & & & & \\
\hline Malestar & 1 & 1 & & & & & & \\
\hline Miedo & 4 & 4 & & & & & & \\
\hline Nerviosismo & 5 & 3 & & & & & & \\
\hline Poco valorada/o & 2 & 2 & & & & & & \\
\hline Preocupación & 2 & 3 & & & & & & \\
\hline RESPONSABLE & 3 & & & & & & & \\
\hline Estrés & 1 & 1 & & & & & & \\
\hline Totales & 63 & 43 & & 77 & 47 & & 55 & 43 \\
\hline \multicolumn{2}{|c|}{ Tristeza } & & \multicolumn{2}{|c|}{ Alegría } & & \multicolumn{3}{|c|}{ Sorpresa } \\
\hline & Inicial & Final & & Inicial & Final & & Inicial & Final \\
\hline Abatimiento & 1 & 1 & AGRADECIDA & 1 & & CURIOSA & 1 & \\
\hline ABRUMADA & 1 & & Alivio & 1 & 2 & Sorpresa & 2 & 1 \\
\hline Agobio & 2 & 2 & Compasión & 1 & 2 & EXPECTACION & & 1 \\
\hline Contrariedad & 1 & 2 & Comprendida & 1 & 4 & & & \\
\hline Desánimo & 11 & 1 & FIRMEZA & 1 & & & & \\
\hline Decepción & 2 & 2 & Seguridad & 1 & 1 & & & \\
\hline Desconcierto & 2 & 8 & Ternura & 1 & 1 & & & \\
\hline Desconfianza & 5 & 1 & ACEPTACIÓN & & 4 & & & \\
\hline Desilusión & 1 & 1 & ADAPTACIÓN & & 1 & & & \\
\hline Herida & 4 & 1 & ANIMADA & & 1 & & & \\
\hline Incomprendido & 1 & 6 & APOYADA & & 1 & & & \\
\hline Incomunicación & 5 & 1 & AUTOESTIMA & & 1 & & & \\
\hline Pena & 1 & 2 & CAPACIDAD & & 2 & & & \\
\hline Pesadumbre & 7 & 1 & COMPROMISO & & 2 & & & \\
\hline Tristeza & 2 & 2 & CONFIANZA & & 1 & & & \\
\hline Zozobra & 3 & 8 & CONFORTADA & & 1 & & & \\
\hline CANSANCIO & & 2 & EMPATIA & & 1 & & & \\
\hline Pereza & 3 & 2 & FUERZA & & 1 & & & \\
\hline Sobrecarga & 3 & 1 & ILUSION & & 1 & & & \\
\hline PESIMISMO & 1 & & AYUDADA & & 2 & & & \\
\hline Hastío & 1 & 1 & SATISFACCIÓN & & 3 & & & \\
\hline & & & SERENIDAD & & 2 & & & \\
\hline & & & TRANQUILIDAD & & 3 & & & \\
\hline Totales & 56 & 46 & & 7 & 37 & & 3 & 2 \\
\hline
\end{tabular}

Todos los adjetivos van precedidos de "sentir" o "sentirse". En mayúsculas las emociones que solo se expresaron al inicio (van también en negrita) o solo al "volver a pensar" (mayúsculas sin negrita). 
Podemos describir como ejemplo las emociones ante uno de los casos presentados y que clasificamos en el apartado de "cuestionamiento de la competencia profesional".

El primer paso era describir el caso: se trataría de una paciente de 36 años, Susana, cuya situación es presentada por su médica al grupo como sigue: "vino a mi consulta Susana, después de haber consultado durante varios años, con distintos síntomas que yo consideraba de ansiedad reactiva en relación a problemas familiares y profesionales. En estas ocasiones le había apoyado emocionalmente y muchas veces le había sugerido que sus malestares físicos pudieran ser manifestaciones de sus tensiones internas. La paciente en las últimas consultas y en esta ocasión solo me demanda recetas que le han prescrito otros especialistas a los que ha acudido por consultas privadas (otorrinolaringólogo, neurólogo, digestólogo)".
El segundo paso era describir las emociones que le surgieron a la profesional ante ello, y fueron: "frustración con puntuación de 8 , sobrecarga con 8 , fastidio e impotencia".

Las emociones surgidas antes y después de "volver a pensar", en todos los casos presentados las hemos agrupado en 5 emociones básicas (los 5 casos de "sorpresa" se han añadido a los de "alegría”), y pueden verse en Fig. 1. La intensidad de las emociones básicas cambia de forma significativa tras "el volver a pensar" $(p<0,001)$.

Los pensamientos irracionales se presentan agrupados en la tabla 3. Después de "volver a pensar" se anotaron los nuevos pensamientos y su síntesis se recoge en tabla 4, donde se aprecia que una mayoría estaban relacionados con la necesidad de mejorar la comunicación, y otros con refutaciones estructuradas a las distorsiones cognitivas que subyacían en los pensamientos iniciales.

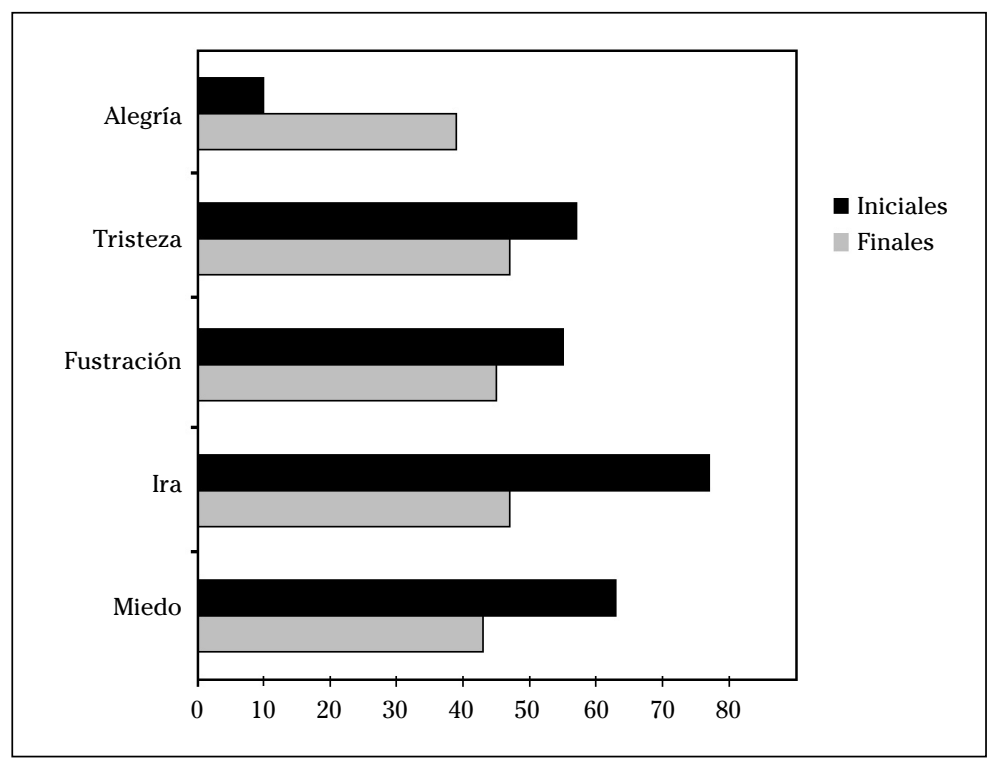

Se han encontrado diferencias significativas en emociones iniciales y finales $\mathrm{p}<0,001$. Se han incluido dentro de alegría, para mejor visualización del gráfico y análisis estadístico, las 2 emociones iniciales y 3 finales de "sorpresa".

Figura 1. Emociones antes (iniciales) y después (finales) de "volver a pensar". 
Tabla 3. Distorsiones cognitivas deducidas de los pensamientos irracionales escritos en ficha 1

\begin{tabular}{|c|c|c|c|}
\hline Nombre de la distorsión & Definición & Número & $\%$ \\
\hline Razonamiento emocional & Usted supone que las cosas son de la forma que usted las siente. & 38 & 31 \\
\hline Falacias de control & $\begin{array}{l}\text { Usted siente que tiene una responsabilidad total de todo o todos, } \\
\text { o bien siente que no tiene. }\end{array}$ & 20 & 16 \\
\hline Filtrado & $\begin{array}{l}\text { Usted presta atención selectivamente a lo negativo y desatiende } \\
\text { lo positivo. }\end{array}$ & 18 & 15 \\
\hline Hipergeneralización & $\begin{array}{l}\text { A partir de un hecho aislado, usted hace una regla general y uni- } \\
\text { versal. Si fracasó una vez, siempre fracasará. }\end{array}$ & 15 & 12 \\
\hline Designación global & $\begin{array}{l}\text { Usted utiliza automáticamente denominaciones peyorativas para des- } \\
\text { cribirse a sí mismo, en vez de describir con exactitud sus cualidades. }\end{array}$ & 13 & 10 \\
\hline Pensamiento polarizado & $\begin{array}{l}\text { Usted lleva las cosas a sus extremos, las expresa en categoría } \\
\text { blanco o negro, sin un término medio. Tiene que ser perfecto o, } \\
\text { de lo contrario, no vale nada. }\end{array}$ & 6 & 5 \\
\hline Autoacusación & $\begin{array}{l}\text { Usted se culpa permanentemente de cosas que pueden no ser } \\
\text { culpa suya. }\end{array}$ & 6 & 5 \\
\hline Personalización & $\begin{array}{l}\text { Usted supone que todo tiene algo que ver con usted y se compara } \\
\text { negativamente con todos los demás. }\end{array}$ & 5 & 4 \\
\hline Lectura de la mente & $\begin{array}{l}\text { Usted supone que no gusta a los demás, no se interesan por usted } \\
\text { etc. sin evidencia real de que sus suposiciones sean correctas. }\end{array}$ & 3 & 3 \\
\hline
\end{tabular}

Fuente de las definiciones: Autoestima. Evaluación y mejora. Matthew McKay y Patrick Fanning ${ }^{24}$. (Pág 67)

Tabla 4. Pensamientos elaborados después de "volver a pensar". Pautas explicativas constructivas

\begin{tabular}{|c|c|}
\hline Elaboración de pensamiento en relación a & $\begin{array}{c}\text { Número } \\
\text { pensamientos }\end{array}$ \\
\hline Reconocer la necesidad de mejorar la comunicación. & 97 \\
\hline Reconocer un compromiso ético y con la profesión. & 10 \\
\hline Reconocer la necesidad de formación. & 10 \\
\hline Refutar la distorsión de "designación global" y para ello "Reconocer cualidades". & 7 \\
\hline Reconocer la necesidad de conectar con la familia. & 5 \\
\hline Refutar la distorsión de "pensamiento polarizado" y para ello "Aceptar las propias limitaciones". & 5 \\
\hline Refutar la distorsión de "filtrado" y para ello "Atender a lo positivo". & 4 \\
\hline Refutar la distorsión de "personalización" y para ello "No compararse". & 2 \\
\hline Refutar la distorsión de "hipergeneralización". & 1 \\
\hline Refutar la distorsión de "lectura de la mente" y para ello "No suponer lo que otros piensan". & 1 \\
\hline Refutar la distorsión de "falacias de control" y para ello "Responsabilidad compartida". & 1 \\
\hline $\begin{array}{l}\text { Refutar la distorsión de "razonamiento emocional" y para ello ver que "No todo es según usted } \\
\text { lo piensa". }\end{array}$ & 1 \\
\hline
\end{tabular}

En el caso de Susana, su médica después de darse cuenta de las emociones que le generó en la consulta antes descrita, realizó el tercer paso, de analizar los pensamientos irracionales que creía ha- bían provocado esas emociones y anotó en ficha 1 lo siguiente: "me tiene en cuenta como profesional pero recurre a privados (y luego viene a por la receta); creo que le estoy dedicando demasiado tiempo y que 
ella marca los tiempos". La médica después de "volver a pensar" (cuarto paso) anotó: "Está tan agobiada... que necesita saber que hay cosas más serias. Su agobio familiar le está repercutiendo en su vida social, le genera inseguridad, quizás le reforzaría más el contactar con la enfermera y que le ayudase con pautas en el cuidado de su familia enferma, o también puedo sugerirle una consulta con psicólogo". Por último (quinto paso) la médica trató de describir las emociones que le surgían ahora y anotó: "Comprensión, adaptación, le estoy ayudando y frustración de intensidad 2".

Podemos decir que en el caso presentado, el pensamiento irracional que le surgió a la profesional fue una de las distorsiones cognitivas descritas por McKay y Fanning ${ }^{24}$ : la de "falacia de control". Después de volver a pensar la médica vio que podía compartir su responsabilidad con la enfermera y psicóloga, y por tanto el pensamiento cambió hacía una pauta explicativa constructiva de "responsabilidad compartida". Las emociones negativas también cambiaron y se rebajaron en intensidad.

El grupo realizó distintas aportaciones a cada caso y éstas se recogen resumidas en tabla 5. En los casos presentados había disminuido más la intensidad de las emociones negativas después de haberlo presentado en el grupo.

En el caso de Susana, su médica anotó que el grupo le había aportado lo siguiente: "puedes utilizar técnicas de comunicación y en la próxima visita devolverle lo que tu piensas de ella, es decir que viene a contarte continuamente las atenciones de otros profesionales y que te gustaría que aparte de esto te cuente cómo se siente y cómo le podrías ayudar", "emplear también otras técnicas de comunicación como escucha activa, o reatribución de síntomas", "ayudar a Susana a ser dueña de su vida y no sólo ser la cuidadora de su familia", "pensar en la hipótesis de que Susana esté acusando que le falla la madre y en etapa de pasar de ser cuidada a ser ella la cuidadora".

Las encuestas utilizadas para valorar el desgaste profesional (MBI) en 2010 y 2012 no presentan diferencias significativas $(\mathrm{p}>0,05)$. Los valores medios en 2010 y 2012 para can- sancio emocional fueron de 14,4 y 13,5 respectivamente; para despersonalización 6,5 y 6,4; y de realización personal 42,8 y 42,4.

Las respuestas a la encuesta diseñada para la evaluación con la mediana de cada item en las respuestas del 2010 y en el 2012 pueden verse en tabla 1 y no se encontraron diferencias significativas $(p>0,05)$. Es decir que las respuestas emocionales provocadas por las 13 situaciones distintas que se plantearon en la encuesta, no fueron diferentes en 2010 y 2012.

\section{DISCUSIÓN}

Este estudio aporta una forma novedosa de analizar la relación profesional sanitario-paciente en los encuentros difíciles. No hemos encontrado otros trabajos similares con los que poder comparar nuestros resultados.

El haber realizado una agrupación de los casos bajo siete epígrafes, puede ampliar o reducir alguna de las problemáticas presentadas, pero se ha realizado intentando incorporar la diversidad y facilitar el análisis. Por otro lado aunque el número de casos trabajados no es muy grande, el hecho de hacerlo un grupo de profesionales con experiencia previa en grupo de reflexión, en un clima de confianza y seguridad, permitió realizar muchas observaciones, recogiendo un amplio número de interesantes aportaciones.

Invita a la reflexión la distribución temática de los encuentros difíciles:

- El $42 \%$ de los temas de estos encuentros corresponden al sentimiento del profesional de que el paciente realiza solicitudes inapropiadas (bajas laborales, recetas...), lo que quizás habla de las distintas expectativas que tienen los pacientes y los profesionales. Esto ya ha sido puesto de manifiesto en diversos estudios ${ }^{26,27}$ en los que encuentran que existe cierta discordancia entre los considerados encuentros difíciles, por los médicos y por los pacientes.

- Un 20\% de casos corresponden a aquellos en los que el profesional siente que se cuestiona su competencia profesional. Ello pudiera guar- 
dar relación con las concepciones que pueden tener los profesionales sobre su propio rol, concebido a veces como de "salvador",2, en un marco paternalista de la relación médico-paciente ${ }^{29}$.

- Los otros casos, tenían relación con dilemas éticos, y malas noticias, que en sí mismos suponen una dificultad añadida al trabajo técnico-resolutivo del profesional en la consulta, como se conoce por otras referencias ${ }^{19,30}$.

Cabe reseñar la disminución significativa en el tipo de emociones negativas antes y después de la reflexión individual del caso y su escritura reflexiva, más allá del simple registro tal y como reflejan otros autores ${ }^{30}$. Se aprecia que algunas de las emociones negativas relacionadas con las básicas de "ira", "miedo", "tristeza" y "disgusto", son más numerosas al inicio. Las emociones relacionadas con "alegría" aparecen con más frecuencia al volver a pensar, reflejando el aspecto positivo de la introspección. La disminución significativa en el tipo de emociones negativas antes y después de la reflexión individual del caso y su escritura reflexiva, parece dar la razón a diversos autores ${ }^{23,26,30}$ que afirman que los sentimientos negativos provocados por algunos encuentros difíciles, no deben ser negados sino reconocidos y elaborados.

Reflexionar individualmente sobre los pensamientos irracionales, ayudó a rebajar la intensidad de las emociones negativas. Hemos clasificado los pensamientos irracionales según las distorsiones cognitivas descritas en la literatura ${ }^{24}$. Especialmente numerosas han sido las distorsiones cognitivas referidas a "razonamiento emocional", que parece indicar la creencia por parte del profesional de que el paciente no puede sentir las cosas de forma diferente a él. Las "falacias de control" también fueron numerosas, en el sentido de que el profesional a veces siente que tiene la responsabilidad de todo como ha sido objetivado en diversos estudios ${ }^{2,28,29}$.

Hacer conscientes las dificultades comunicacionales, tras "volver a pensar", parece poner de manifiesto la complejidad de la comunicación, que va más allá de unas simples técnicas 9 . Que en 22 casos se haya tratado de refutar la distorsión cognitiva, al "volver a pensar", sobre todo con distorsiones de "pensamiento polarizado", y de "designación global", que han pasado a pautas explicativas constructivas (como pensamientos de "aceptar las limitaciones", a "reconocer cualidades"), parece evidenciar la utilidad de la técnica de refutación para el manejo de emociones negativas, y cómo estas se rebajan con estas pautas.

Parece difícil objetivar si este grupo de reflexión contribuyó a mejorar el desgaste profesional, porque éste ya era bajo en la evaluación de 2010, encontrando resultados mejores que la media hallada en el ámbito sanitario, en el que para cansancio emocional suelen referir valores medios 19-26, para despersonalización 6-9 y para realización personal entre $34-39^{25,31}$. En otro estudio reciente con médicos de familia ${ }^{32}$, más del $37 \%$ de los médicos tienen puntuaciones de burnout altas en cada una de las dimensiones. En nuestro trabajo ningún profesional tiene puntuaciones altas y se obtienen mejores puntuaciones que las encontradas en profesionales que pertenecen a grupos de comunicación y salud ${ }^{33}$. A ello puede contribuir que las personas que contestan el test MBI en el 2010 ya llevaban al menos 2 años trabajando en estos temas en grupo de reflexión ${ }^{19}$.

La encuesta de elaboración propia (Tabla 1) midió la autopercepción de los sentimientos que suscitan los encuentros difíciles y estos no cambiaron del $2010 \mathrm{al} 2012$. No sabemos si sus capacidades para el manejo de las situaciones cambiaron, ya que no hemos encontrado un instrumento válido para poder evaluar las capacidades adquiridas en el manejo de los sentimientos, después de los 2 años de trabajo en el grupo de reflexión. Sólo podemos remitirnos a la evaluación satisfactoria que los componentes del grupo hacen sobre su participación en el mismo, y a los comentarios de cada participante que al final de cada caso presentado quedaban escritos en la ficha (Tabla 5).

El grupo de reflexión parece constatarse como una estrategia de ayuda para los profesionales sanitarios, en base a una mejor comprensión tanto de las emociones que surgen en la consulta en los encuentros 
difíciles, como de los pensamientos irracionales que se asocian a las mismas. Esto ya ha sido descrito en otros estudios ${ }^{2,18,19}$, y se evidencia en el presente, tanto en la evaluación positiva que realizó el grupo tras la experiencia, como en el balance positivo que se describe de las aportaciones del grupo como tal (Tabla 5). Destaca que en más de la mitad de los casos el profesional sanitario especificaba que el grupo, le aportó sugerencias en el plan de actuación, o rebajó sus emociones negativas, o le ayudó a la reflexión sobre distintos aspectos.

A tenor de los resultados y discusión expuestos, y para próximas experiencias, puede ser de interés el realizar la presentación de casos de manera sistemática, de manera que todos los estilos de entrevista y variedades profesionales estuvieran igualmente representados para aportar mayor riqueza y reducción de posibles sesgos.

Como sugieren diversos autores ${ }^{27,28,30}$, es importante el autocuidado del profesional. Reconocer las emociones, así como las distorsiones cognitivas contribuye a poder aceptarlas, analizarlas y reconducirlas. Esto es una forma de entender mejor lo que está sucediendo en la relación médico-paciente y a poder mostrar empatía sobre todo en los encuentros difíciles. De esta forma el reconocimiento y análisis de las emociones conlleva un menor coste emocional para el profesional sanitario y ayuda a mejorar la relación terapéutica con el paciente.

La metodología cognitivo-conductual, como ha sido puesto de manifiesto por algunos autores ${ }^{34}$, tiene sus limitaciones al no estar basada en un análisis en profundidad de los diferentes fenómenos que puede suceder en una consulta, como son, por ejemplo, las diferentes expectativas de profesionales y pacientes, el poder, el clima, las asunciones de los profesionales y los consultantes, la capacidad de introspección o reflexión sobre uno mismo, o la empatía. Esto se ha subsanado en parte al comentar los casos en el grupo de reflexión. Algunos de los aspectos de ayuda explicitados (Tabla 5), han sido: entender al paciente o a los familiares y entender mejor el suceso.

Tabla 5. Aportaciones del grupo a los casos presentados

\begin{tabular}{lc}
\multicolumn{1}{c}{ Tipo de aportación especificada } & $\begin{array}{c}\text { No casos en que } \\
\text { se aportaba }\end{array}$ \\
\hline $\begin{array}{l}\text { Sugerencias en el plan de actuación (en } 2 \text { casos se concreta más: en cuanto a pautas frente } \\
\text { a bajas laborales). }\end{array}$ & 14 \\
\hline $\begin{array}{l}\text { Ver que hay otros casos similares (alguno remarca caso de difícil diagnóstico y sin cambios } \\
\text { en pronóstico). }\end{array}$ & 8 \\
\hline $\begin{array}{l}\text { Ha rebajado mucho mis emociones negativas(tristeza, frustración, culpa... y en un caso "me } \\
\text { ayudó a explicar porque no se rebajó la intensidad de las emociones"). }\end{array}$ & 7 \\
\hline Ayuda a entender al paciente o los familiares. & 6 \\
\hline Reflexión que me ayuda a entender mejor el suceso. & 3 \\
\hline Reflexión que me ayuda a pensar sobre los dilemas éticos del caso. & 3 \\
\hline Remarcar las actuaciones acertadas, o no acertadas. & 3 \\
\hline $\begin{array}{l}\text { Surgen sentimientos positivos ante el grupo (agradecimiento por escucha, compresión, } \\
\text { aportación, apoyado, cuestionado...). }\end{array}$ & 2 \\
\hline $\begin{array}{l}\text { Rectificar distorsiones cognitivas (en un caso del "pensamientos polarizado" y ver que "no } \\
\text { soy pluscuamperfecta" y en otro de la de "falacias de control” y "ver la necesidad de que la } \\
\text { dirección se implique"). }\end{array}$ & 2 \\
\hline $\begin{array}{l}\text { Necesidad de mejorar la comunicación (en 1 caso en cuanto a trasmitir la información y en } \\
\text { otro hacer contrabalanceo). }\end{array}$ & \\
\hline
\end{tabular}


Podemos concluir que la experiencia de grupo de reflexión y la metodología cognitivo-conductual descrita en este estudio, al emplearse conjuntamente, se complementan y posibilitan el trabajo en grupo sin precisar un conductor profesional. Algunos autores ${ }^{35}$ defienden que la reestructuración cognitiva acentúa la evaluación realista de las situaciones difíciles y sirve para reducir la probabilidad de desarrollo de burnout y depresión.

Agradecimientos: Queremos agradecer a todos los compañeros/as que han participado en el grupo de reflexión, sin los cuales no hubiera sido posible la realización de este trabajo.

\section{BIBLIOGRAFÍA}

1. GAMINDE I. La interacción en el sistema sanitario: médicos y pacientes. Informe SESPAS 2002; 5: 67-80.

2. Ruiz Moral R, Rodríguez JJ, Epstein R. ¿Qué estilo de consulta debería emplear con mis pacientes?: reflexiones prácticas sobre la relación médico-paciente. Aten Primaria 2003; 32: 594-602.

3. Borrell-Carrio F, Suchman Al, Epstein R. The Biopsychosocial model 25 years later: principles, practice and scientific inquiry. Ann Fam Med 2004; 2: 576-582.

4. Bellón JA, Delgado A, Luna J, Lardeli-Claret P. Patient psychosocial factors and primary care consultation: a cohort study. Fam Pract 2007; 24: 562-569.

5. Deleda G, Moretti F, Rimondini M, Zimmerman C. How patients want their doctor to communicate. A literature review on primary care patients' perspective. Patient Educ Couns 2013; 90: 297-306.

6. Little P. Preferences of patients for patient centred approach to consultation in primary care: observational study. BMJ 2001; 32: 468475.

7. Koekroek B,van Meijel B, van Ommen J, Pennings $\mathrm{R}$, KaASENBROOD A, HutschemaEKers $\mathrm{G}$ et al. Ambivalent connections: a qualitative study of the care experiences of non-psychotic chronic patients who are perceived as "difficult" by professionals. BMC Psychiatry 2010; 10: 96-103.

8. Ruiz Moral R, Peralta M, Pérula la, Olloqu J, Carrión de la Fuente T, Sobrino A et al. Opiniones y percepciones de los pacientes sobre su participación en la toma de decisiones en las consultas de medicina de familia. Aten Primaria 2012; 44: 5-12.

9. Zoppi K, Epstein RM. ¿Es la comunicación una habilidad? Las habilidades comunicativas para mantener una buena relación. An Sist Sanit Navar 2001; 24 (Suppl 2): 23S-31S.

10. Amezcua M, Gálvez A. Los modos de análisis en investigación cualitativa en salud: Perspectiva crítica y reflexiones en voz alta. Rev Esp Salud Pública 2002; 76: 423-436.

11. Prieto Ma, Danet A, Escudero MJ, Ruiz azarola A, Pérez Corral O, García Toyos N. Definición de competencia médica según pacientes crónicos del sistema sanitario público de Andalucía. Gac Sanit 2012; 26: 450-456.

12. Arillo A, Vilches C, Mayor M, Gurpegui JR, Arroyo C, Estremera V. Pacientes hiperfrecuentadores y difíciles: ¿cómo se sienten atendidos por sus médicos? An Sist Sanit Navar 2006; 29: 33-44.

13. Girón M, Beviá B, Medina E, Simón M. Calidad de la relación médico paciente y resultados de los encuentros clínicos en Atención primaria de Alicante: un estudio con grupos focales. Rev Esp Salud Pública 2002; 76: 561-575.

14. Tzzón JL. Sobre los grupos Balint, el movimiento Balint y el cuidado de la relación médico paciente. Aten Primaria 2005; 36: 453-555.

15. Shorer Y, Biderman A, Levy A, Rabin S, Kami A, MAoz B et al. Family Physicians leaving their clinic- The Balint group as an opportunity to say good-bye. Ann Fam Med 2011; 9: 549-551.

16. LICHTENSTEIN A. Integrating intuition and reasoning. How Balint groups can help medical decision making. Aust Fam Physician 2006; 35: 987-989.

17. Tizón JL. Grupos de reflexión en Atención Primaria. I. Su origen. Aten Primaria 1993; 11: 309-312.

18. Tizón JL. Grupos de reflexión en Atención Primaria. II. Algunos elementos teóricos y técnicos. Aten Primaria 1993; 11 361-366.

19. Arillo A, Zabalegui MJ, Ayarra M, Fuertes C, LoAYSSA JR, PAScual P. El grupo de reflexión como una herramienta para mejorar la satisfacción y desarrollar la capacidad introspectiva de los profesionales sanitarios. Aten Primaria 2009; 41: 688-694.

20. Seligman M. La auténtica felicidad. Ed. Barcelona: Vergara S.A., 2003.

21. BimBela JL. Gimnasia emocional. Pasamos a la acción. Monogr EASP 2008; 46.

22. Ekman P, Davidson RJ, Friesen WV. Duchenne's smile: Emotional expression and brain physiology II. J Pers Soc Psychol 1990; 58: 342-353. 
23. Tracy JL, Robins, RW, Schriber RA. Development of a FACS-verified set of basic and self-conscious emotion expressions. Emotion2009; 4: 554-559.

24. McKay M, FAnNing P. Autoestima. Evaluación y mejora. Ed.Barcelona: Martínez Roca, 1991.

25. MASLACH C, JACKSON SE. Maslach Burnout Inventory. En: Seisdedos N, editor. Manual del Inventario Burnout de Maslach: síndrome del "quemado" por estrés asistencial. Ed. Madrid: TEA S.A. 1997; p. 5-28.

26. Gulbrandsen P, Benth JS, Dahl FA, Jens BF, Finset A, Hall JA. Specialist physicians' sensitivity to patient affect and satisfaction. Med Care 2012; 50: 290-293.

27. Mas X, Navarro M, Vázquez J, Delso C, Mahfouz T, Almeda J. "Ecuentros difíciles" en consultas de atención primaria: una perspectiva de pacientes y médicos. Aten Primaria 2009; 41: 9-17.

28. Loayssa JR, Ruiz R, García Campayo J. ¿Por qué algunos médicos se vuelven poco éticos (¿malvados?) con sus pacientes? Aten Primaria 2009; 41: 646-649.

29. Jiménez-De Gracia, Ruiz-Moral R, Gavilán E, Hueso C, Cano D, Alba MA. Opiniones de los médicos de familia acerca de la implicación de los pacientes en la toma de decisiones: un estudio con grupos focales. Aten Primaria 2012; 44: 379-386.

30. Benito Oliver E, Arranz Carrillo de Albornoz P, CANCIO López H. Herramientas para el autocuidado del profesional que atiende a personas que sufren. FMC 2011; 18: 59-65.

31. Cebrià J, Segura J, Corbella S, Sos P, Comas O, García $\mathrm{M}$ et al. Rasgos de personalidad y burnout en médicos de familia. Aten Primaria 2001; 27: 39-52.

32. Matía AC, Cordero J, Mediavilla JJ, Pereda MJ, GonzÁlez ML, GonzÁlEz A. Evolución del burnout y variables asociadas en los médicos de atención primaria. Aten Primaria 2012; 44: 532-539.

33. Cebria J, Palma C, Segura J, Gracia R, Pérez SánCHEz J. Entrenamiento en habilidades comunicacionales podría ser un factor preventivo del síndrome de burnout en médicos de familia. Rev Psiquiatría Fac Med. Barna 2006; 33: 34-40.

34. Freeling P, M. Harris C. The doctor-patient relationship. Ed. Livingstone: Churchill, 1967.

35. Mészáros V, Cserháti Z, Oláh A, Perczel Forintos D, ADÁm S. Coping with work-related stress in health care professionals - strategies for prevention of burnout and depression. Orv Hetil 2013; 154: 449-454.

\section{ANEXO 1 \\ Plantilla recogida de casos \\ Registro de emociones y pensamientos irracionales asociados \\ Situación o acontecimiento difícil}

Describe la situación seleccionada

\begin{tabular}{|l|c|c|c|c|}
\hline $\begin{array}{c}\text { Emoción inicial } \\
\text { (cuantificar de 1 a 10) }\end{array}$ & $\begin{array}{c}\text { Pensamiento } \\
\text { irracional asociado }\end{array}$ & $\begin{array}{c}\text { Volver } \\
\text { a pensar }\end{array}$ & $\begin{array}{c}\text { Emoción posterior } \\
\text { (cuantificar de 1 a 10) }\end{array}$ & $\begin{array}{c}\text { Actuación } \\
\text { (realizada o planificada) }\end{array}$ \\
\hline & & & & \\
& & & &
\end{tabular}

Aportaciones del grupo: (cuantifica de nuevo, en la medida de lo posible, los cambios en las emociones, y las aportaciones en general). 